\title{
Adiponectin Attenuates the Inflammation in Atopic Dermatitis-Like Reconstructed Human Epidermis
}

\author{
Hee-Seok Seo, Ki Hyun Seong, Chang-Deok Kim ${ }^{1}$, Seong Jun Seo ${ }^{2}$, Byung Cheol Park, \\ Myung Hwa Kim, Seung-Phil Hong \\ Department of Dermatology, Dankook University College of Medicine, Cheonan, ${ }^{1}$ Department of Dermatology, Chungnam National \\ University College of Medicine, Daejeon, ${ }^{2}$ Department of Dermatology, Chung-Ang University Hospital, Seoul, Korea
}

Background: Atopic dermatitis (AD) is a chronic disorder, with a vicious cycle of repetitive inflammation and deterioration of the epidermal barrier function. Adiponectin, an adipokine, has anti-inflammatory effects on various metabolic and inflammatory disorders. Recently, its level was found to be reduced in serum and tissue samples from AD patients. Objective: We aimed to investigate the effects of adiponectin on epidermal inflammation and barrier structures in AD skin. Methods: A three-dimensional in vitro epidermal equivalent model mimicking AD was obtained by adding an inflammatory substance cocktail to normal human epidermal equivalents (HEEs). The expression of epidermal differentiation markers, primary inflammatory mediators, and lipid biosynthetic enzymes was compared between adiponectintreated AD-HEEs, untreated control AD-HEEs, and normal HEEs. Results: Adiponectin co-treatment 1) inhibited the increase in mRNA expression of major inflammatory mediators (carbonic anhydrase II, neuron-specific NEL-like protein 2, thymic stromal lymphopoietin, interleukin-8, tumor necrosis factor-alpha, and human beta-defensin-2) from keratinocytes in AD-inflammatory HEEs, 2) enhanced the expression of lipid biosynthetic enzymes (fatty acid synthase,

Received August 8, 2018, Revised October 21, 2018, Accepted for publication November 5, 2018

Corresponding author: Seung-Phil Hong, Department of Dermatology, Dankook University College of Medicine, 201 Manghyang-ro, Dongnamgu, Cheonan 31116, Korea. Tel: 82-41-550-6485, Fax: 82-41-552-7541, E-mail: zamoo97@naver.com ORCID: https://orcid.org/0000-0002-0684-498X

This is an Open Access article distributed under the terms of the Creative Commons Attribution Non-Commercial License (http://creativecommons. org/licenses/by-nc/4.0) which permits unrestricted non-commercial use, distribution, and reproduction in any medium, provided the original work is properly cited.

Copyright $\odot$ The Korean Dermatological Association and The Korean Society for Investigative Dermatology
HMG CoA reductase, and serine-palmitoyl transferase), and 3) promoted the expression of differentiation factors, especially filaggrin. We also found that the expression of adiponectin receptor-1 and -2 decreased in the epidermis of chronic AD lesion. Conclusion: Activation of the adiponectin pathway is expected to enhance epidermal differentiation and barrier function as well as attenuate inflammatory response to AD as a therapeutic approach. (Ann Dermatol 31(2) 186 195, 2019)

\section{-Keywords-}

Adiponectin, Atopic dermatitis, Epidermal barrier, Keratinocyte, Lipid synthesis

\section{INTRODUCTION}

Atopic dermatitis (AD) is a complex disorder that involves both inflammation and skin barrier dysfunction. Reduced skin barrier function, specifically caused by abnormalities in filaggrin expression and lipid synthesis, can lead to the release of thymic stromal lymphopoietin (TSLP) from keratinocytes, inducing $\mathrm{T}$ helper 2 (Th2) immune response ${ }^{1}$. Subsequently, Th2 cytokines, such as interleukin (IL)-4 and IL-13, and pro-inflammatory cytokines, such as IL-8 and tumor necrosis factor-alpha (TNF- $\alpha$ ), are secreted, resulting in a dermal inflammatory reaction.

Both IL-4 and IL-13 cause abnormalities in lipid production and epidermal differentiation ${ }^{2,3}$ as well as inhibition of antimicrobial peptide synthesis in the epidermis ${ }^{4}$, which allows microbial invasion that is damaging the epidermal barrier. Furthermore, IL-8 (CXCL8), a pro-inflammatory cytokine that directly affects immune cells, including polymorphonuclear cells, is secreted by immune cells after an- 
tigen sensitization ${ }^{5,6}$. Keratinocytes are considered another major source of IL-8 that is overexpressed in AD skin ${ }^{6,7}$, leading to a vicious cycle of repetitive inflammation and deterioration of the barrier function.

Adipocytes and sebocytes secrete adiponectin, an adipokine, which reaches a concentration of $3 \sim 30 \mu \mathrm{g} / \mathrm{ml}$ in the plasma ${ }^{8,9}$. Adiponectin functions as signal molecule via at least two adiponectin receptors, AdipoR1 and AdipoR2, on target cells ${ }^{10}$. Because it acts primarily as a metabolic mediator of insulin sensitivity and glucose homeostasis, adiponectin has been implicated in various diseases, especially metabolic disorders such as diabetes and obesity and cardiovascular diseases ${ }^{10-12}$. It has been demonstrated that the adiponectin level in the plasma is negatively associated with the accumulation of body fat, particularly visceral fat, and that the plasma adiponectin levels are low in obese individuals ${ }^{13}$. In addition, adiponectin plays a role in controlling inflammation during immune responses and, therefore, it has emerged as an important therapeutic target for chronic inflammatory disorders due to its pleiotropic activities in regulating inflammation and immune response, and vascular homeostasis ${ }^{14-16}$. Several studies have reported on the association between psoriasis and adiponectin in relation to inflammation and metabolic abnormalities as well as the possibility to exploit the adiponectin signaling mechanism as a therapeutic target ${ }^{17-19}$.

Recent advances in three-dimensional (3D) skin equivalent models have facilitated the development of AD-like $3 \mathrm{D}$ skin equivalent models by subjecting the cells to a treatment with major cytokines involved in AD pathogenesis $^{6,20,21}$. These models can be used to study the inflammation and barrier function of the epidermis. Specifically, epidermal equivalent models of AD consisting of keratinocytes enable studies that are focused on epidermal physiological phenomena, such as morphogenesis, proliferation, and differentiation and the properties of stratum corneum lipids.

In the present study, we investigated the effects of adiponectin, which has anti-inflammatory and cytoprotective effects, on inflammation, lipid synthesis, and epidermal differentiation in the 3D epidermis equivalent model exposed to the inflammatory condition of AD.

\section{MATERIALS AND METHODS}

\section{Reagents and antibodies}

Recombinant human full-length adiponectin protein was obtained from Biobud (Seongnam, Korea). We obtained antibodies against AdipoR1 and AdipoR2 from Santa Cruz Biotechnology (Santa Cruz, CA, USA); filaggrin from Abcam (Cambridge, UK). Polyinosinic-polycytidylic acid [Poly(l:C)] was purchased from Sigma-Aldrich (St. Louis, MO, USA), and TNF- $\alpha$, IL-4, and IL-13 were from R\&D Systems (Minneapolis, MN, USA).

\section{Immunohistochemical staining}

Subjects were not recruited specifically for this study to obtain human skin samples, but the usage of skin samples from subjects who had previously provided informed consent for research, was approved by the Dankook University Hospital Institutional Review Board (DKUH2015-02-017-001) and the analysis was performed retrospectively.

For examination by light microscopy, full-thickness human skin specimens or epidermal equivalents were fixed in $10 \%$ formalin solution. After dehydration with a graded series of ethanol and xylene, the tissues were embedded in paraffin and cut into $4-\mu \mathrm{m}$ thick sections. After de-paraffinization and rehydration, hematoxylin and eosin (H\&E) stains were used for histological examination.

Standard immunohistochemical (IHC) protocol was followed after de-paraffinization and rehydration. Subsequently, each 4- $\mu \mathrm{m}$ section in $10 \mathrm{mM}$ sodium citrate $(\mathrm{pH} 6.0)$ was heated in a microwave oven for antigen retrieval, after which endogenous peroxidase activity was blocked with blocking serum solution (Dako, Carpinteria, CA, USA). Thereafter, adjacent sections from the same paraffin block were incubated with the primary antibodies: AdipoR1, AdipoR2, and Filaggrin (diluted $1: 100$ ) at $4{ }^{\circ} \mathrm{C}$ overnight, followed by incubation with secondary antibody. Signals were developed with two-component high-sensitivity diaminobenzidine (DAB) chromogenic substrate (Dako Cytomation Liquid DAB Substrate Chromogen System; Dako). The slides were covered with cover glass and then examined using a light microscope (BX43, Olympus, Tokyo, Japan)

\section{The 3D in vitro AD-like human epidermal equivalent model}

The 3D in vitro epidermal equivalent model based on cultured HEEs was exposed to an AD-inducing inflammatory cytokine cocktail to create atopic conditions according to a previously published method (Fig. 1$)^{6,20,21}$. The 3D human epidermal equivalents (HEEs) were supplied by TEGO Science Inc. (Seoul, Korea) on days 9 10 of the experiment. Briefly, to construct epidermal equivalents, keratinocytes were seeded into culture inserts. After culturing the cells for 3 days, the constructs were cultured at the air-liquid interface for 10 days. On day 11 after the air-lift culture of constructs, the inflammatory cocktail $(10 \mu \mathrm{g} / \mathrm{ml}$ Poly(I:C), $3.5 \mathrm{ng} / \mathrm{ml}$ TNF- $\alpha, 30 \mathrm{ng} / \mathrm{ml}$ each IL-4 and IL-13) was supplemented (AD-like model, AD-HEEs) or not supplemented (normal-HEEs) to the culture medium until har- 


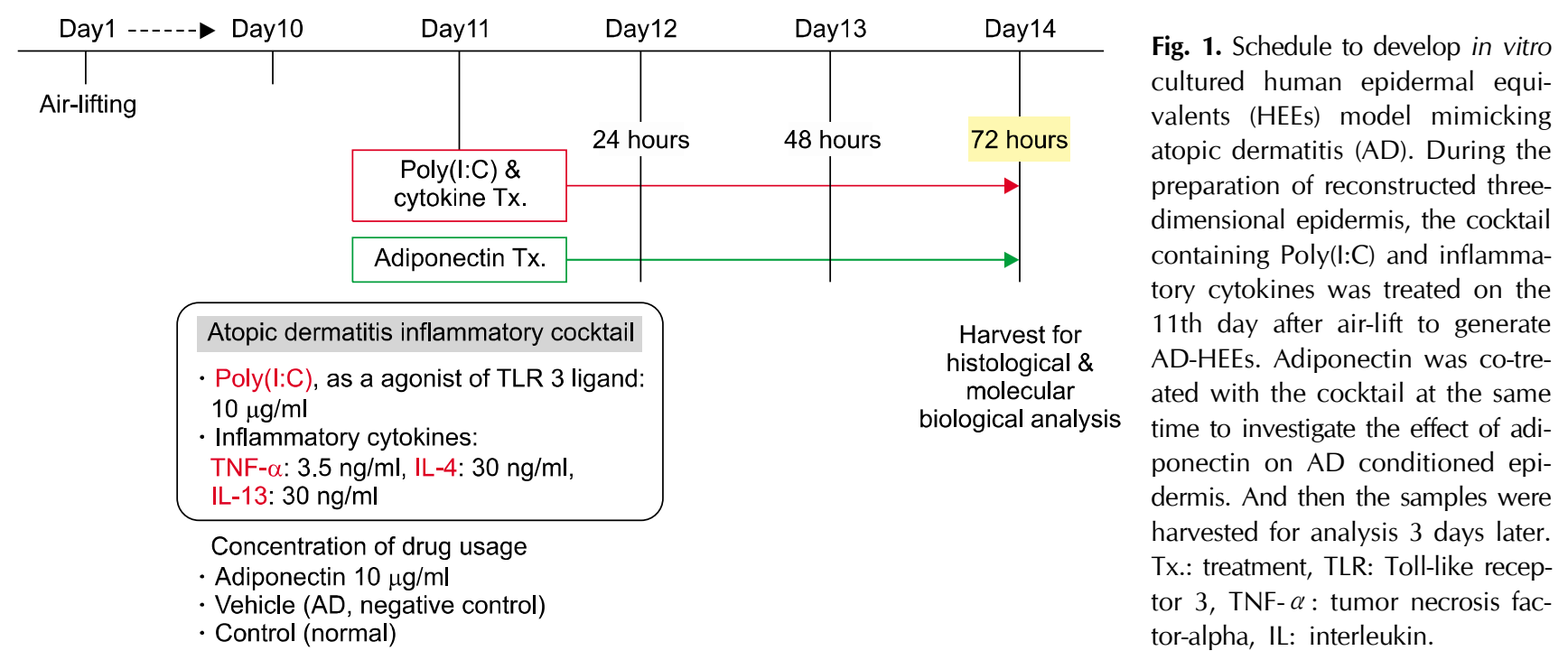

vest after 72 hours on day 14 . The constructs were incubated at $37^{\circ} \mathrm{C}$ and $5 \% \mathrm{CO}_{2}$, and the medium was changed every 2 or 4 days. The culture medium was supplied by TEGO Science Inc.

\section{Quantitative real-time reverse transcriptase polymerase chain reaction analysis}

The total RNA from HEK was extracted using TRIzol ${ }^{\mathbb{R}}$ reagent (Invitrogen, Carlsbad, CA, USA), and the amount of RNA was determined using the Nanodrop 2000 spectrophotometer (Thermo Fisher Scientific, Carlsbad, CA, USA). The total RNA $(1 \mu \mathrm{g})$ was reverse transcribed using M-MLV reverse transcriptase (Promega, Madison, WI, USA) according to the instruction of the manufacturer. The quantitative real-time reverse transcriptase polymerase chain reaction (qRT-PCR) was performed with SYBR Green PCR master mix (Applied Biosystems, Foster City, CA, USA) using Quantstudio 3 (PCR Instrument system, Thermo Scientific Inc., Waltham, MA, USA). The PCR gene expression level was normalized to GAPDH expression. Quantification was performed by the critical threshold (CT) method. The primer sequences for the qRT-PCR are presented in Table 1.

\section{Statistical analyses}

The data are expressed as mean \pm standard error. The data were analyzed by the paired Student's t-test and analysis of variance (ANOVA) with Bonferroni's post hoc correction for comparison between the mean of two numeric values. Statistical significance was defined at $p$-value $<0.05$ and the statistical analyses were performed using PASW Statistics ver. 18.0 (IBM Co., Armonk, NY, USA).

\section{RESULTS}

Expression of adiponectin receptors decreased in the lesions of AD

To investigate the changes in the expression of Adipo $\mathrm{R}$ in the lesional skin of $A D, I H C$ staining was performed with anti-AdipoR1 and AdipoR2 antibodies in three skin samples each from young normal adults and same-aged adults with AD. The expression of AdipoR1 and AdipoR2 was reduced in the lesional epidermis of patients with AD, suggesting that the adiponectin signal transduction activity may be weakened in the AD epidermis (Fig. 2).

\section{Adiponectin treatment reduced AD-specific inflammatory mediators in the inflammatory HEEs with AD features}

By the qRT-PCR analysis, the changes in the expression of major inflammatory mediators in AD were evaluated after adiponectin treatment in the 3D AD-like model. Carbonic anhydrase II (CAII) and neuron-specific NEL-like protein 2 (NELL2) have been reported as epidermal molecular markers of AD owing to their specific up-regulation in the atopic lesional epidermis ${ }^{21,22}$. Therefore, the increase in the expression of CAII and NELL2 in our AD HEEs indicated that it is similar to the epidermis of AD. This increase was subsequently decreased to the control level in the reconstructed epidermis co-treated with adiponectin for 72 hours (Fig. 3A, B). In keratinocytes, the increased expression of two cytokines, TSLP and IL-8, typically secreted during Th2 immune responses, was also significantly normalized after adiponectin treatment (Fig. 3C, D). Moreover, the expression of TNF- $\alpha$, a pro-inflammatory cytokine, and human beta defensin 2, an anti-microbial peptide, was similar to that of TLSP (Fig 3E, F). These results suggested that 
Table 1. Primer sequences for real-time polymerase chain reaction

\begin{tabular}{|c|c|}
\hline Gene & Nucleotide sequence $\left(5^{\prime}-3^{\prime}\right)$ \\
\hline \multicolumn{2}{|l|}{ CAII } \\
\hline Sense & AAC AAT GGT CAT GCT TTC AAC G \\
\hline Antisense & TGT CCA TCA AGT GAA CCC CAG \\
\hline \multicolumn{2}{|l|}{ NELL2 } \\
\hline Sense & TAA GGG TAT AAT GCA AGA TGT CCA ATT \\
\hline Antisense & AGA TCT GGG CAC TGA GCA ATA AA \\
\hline \multicolumn{2}{|r|}{ ( } \\
\hline Sense & CAG GCT ATT CGG AAA CTC A \\
\hline Antisense & GTG CTG TGA AAT ATG ACC A \\
\hline \multicolumn{2}{|r|}{ 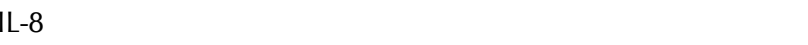 } \\
\hline Sense & TTG GCA GCC TTC CTG ATT \\
\hline Antisense & AAC TTC TCC ACA ACC CTC TG \\
\hline \multicolumn{2}{|l|}{ TNF- $\alpha$} \\
\hline Sense & CAG AGG GCC TGT ACC TCA TC \\
\hline Antisense & GGA AGA CCC CTC CCA GAT AG \\
\hline \multicolumn{2}{|l|}{ hBD2 } \\
\hline Sense & GGA TGA CAT ATG GCT CCA CTC TT \\
\hline Antisense & GAT GCC TCT TCC AGG TGT TTT T \\
\hline \multicolumn{2}{|r|}{ 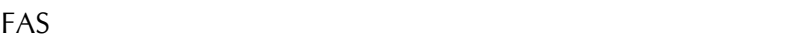 } \\
\hline Sense & TCG TGC AGG TGC TTG CGG AG \\
\hline Antisense & GCC GAA GCC ACC CAG ACC AC \\
\hline \multicolumn{2}{|r|}{ 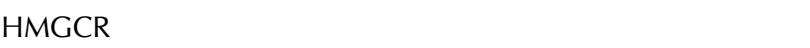 } \\
\hline Sense & ACG GTG GGT GGT GGG ACC AA \\
\hline Antisense & TCC TGC TGC CAA TGC TGC CA \\
\hline \multicolumn{2}{|r|}{ 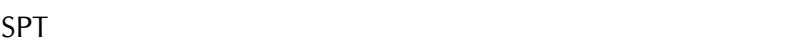 } \\
\hline Sense & AGT GGG TTC TGG TGG AGA TG \\
\hline Antisense & TTT GGG ACA GGA GGA ACA AG \\
\hline \multicolumn{2}{|l|}{ SREBP1a } \\
\hline Sense & GCT GCT GAC CGA CAT CGA A \\
\hline Antisense & ATG TGG CAG GAG GTG GAT AC \\
\hline \multicolumn{2}{|l|}{ SREBP1C } \\
\hline Sense & GGA GCC ATG GAT TGC ACT TT \\
\hline Antisense & ATG TGG CAG GAG GTG GAT AC \\
\hline \multicolumn{2}{|l|}{ SREBP2 } \\
\hline Sense & TGG СТT СТС ТСС СТА СТС СА \\
\hline Antisense & GAG AGG CAC AGG AAG GTG AG \\
\hline \multicolumn{2}{|l|}{ Filaggrin } \\
\hline Sense & GGA TCC TCT CAC CGC GAT AC \\
\hline Antisense & GCC TTT CAG TGC CCT CAG AT \\
\hline \multicolumn{2}{|l|}{ Involucrin } \\
\hline Sense & GAT GTC CCA GCA ACA CAC AC \\
\hline Antisense & TGC TCA CAT TCT TGC TCA GG \\
\hline \multicolumn{2}{|l|}{ Loricrin } \\
\hline Sense & GGA GTT GGA GGT GTT TTC CA \\
\hline Antisense & ACT GGG GTT GGG AGG TAG TT \\
\hline \multicolumn{2}{|l|}{ GAPDH } \\
\hline Sense & CGG ATT TGG TCG TAT TGG G \\
\hline Antisense & CTC GCT CCT GGA AGA TGG \\
\hline
\end{tabular}

CAII: carbonic anhydrase II, NELL2: neuron-specific NEL-like protein 2, TSLP: thymic stromal lymphopoietin, IL: interleukin, TNF- $\alpha$ : tumor necrosis factor-alpha, hBD2: human beta defensin 2, FAS: fatty acid synthase, HMGCR: HMG CoA reductase, SPT: serine-palmitoyl transferase, SREBP: sterol regulatory binding protein. adiponectin has the effect of counteracting the inflammatory responses of AD.

\section{Adiponectin reduced the histological inflammatory response}

In the H\&E-stained sample used to evaluate the degree of morphological changes, the AD-HEE group (treated with AD cocktail only) showed intercellular spongiosis, and lack of keratohyalin granules and stratum corneum formation (Fig. 4B). In contrast, the adiponectin-treated AD-HEEs demonstrated less spongiosis and relatively well-developed corneal layer with basket-weave structure (Fig. 4C), and they stabilized to a relatively normal epidermis (normal-HEEs) (Fig. 4A).

\section{Adiponectin increased the expression of lipogenic enzymes and transcription factors in AD-like inflam- matory conditions}

We further evaluated the epidermal lipid synthetic potential closely related to skin barrier function. The expression of the rate-limiting enzymes for fatty acid, cholesterol, and ceramide biosynthesis (fatty acid synthase [FAS], HMG CoA reductase [HMGCR], and serine palmitoyl transferase [SPT], respectively) was decreased in AD-HEEs but significantly upregulated by adiponectin treatment (Fig. 5A C). In addition, the mRNA expression of the transcription factors encoding the genes related to the biosynthesis of fatty acid or cholesterol, sterol regulatory element binding protein-1c (SREBP1C), and -2 (SREBP2), was restored to normal control level in adiponectin-co treated AD-HEEs (Fig. $5 D \sim F)$. These results suggested that the lipid synthesis ability of reconstructed epidermis, which was reduced in the AD condition, can be restored by the adiponectin effect.

\section{Adiponectin potentiated the epidermal differentiation markers in the inflammatory state of AD}

The mRNA expression of filaggrin, involucrin, and loricrin, the major epidermal differentiation proteins, was significantly decreased due to AD inflammation, but the expression was increased above the normal control level after adiponectin treatment (Fig. 6A C). Further, the expression of filaggrin protein in HEEs, which is important for the pathogenesis of $A D$, was further examined by immunohistochemistry. Adiponectin-treated AD-HEEs demonstrated the increased expression of filaggrin in the viable layer of epidermal equivalent compared with that in AD-HEEs (Fig. 6D). Thus, adiponectin might control epidermal differentiation abnormalities under AD inflammatory conditions. 
A

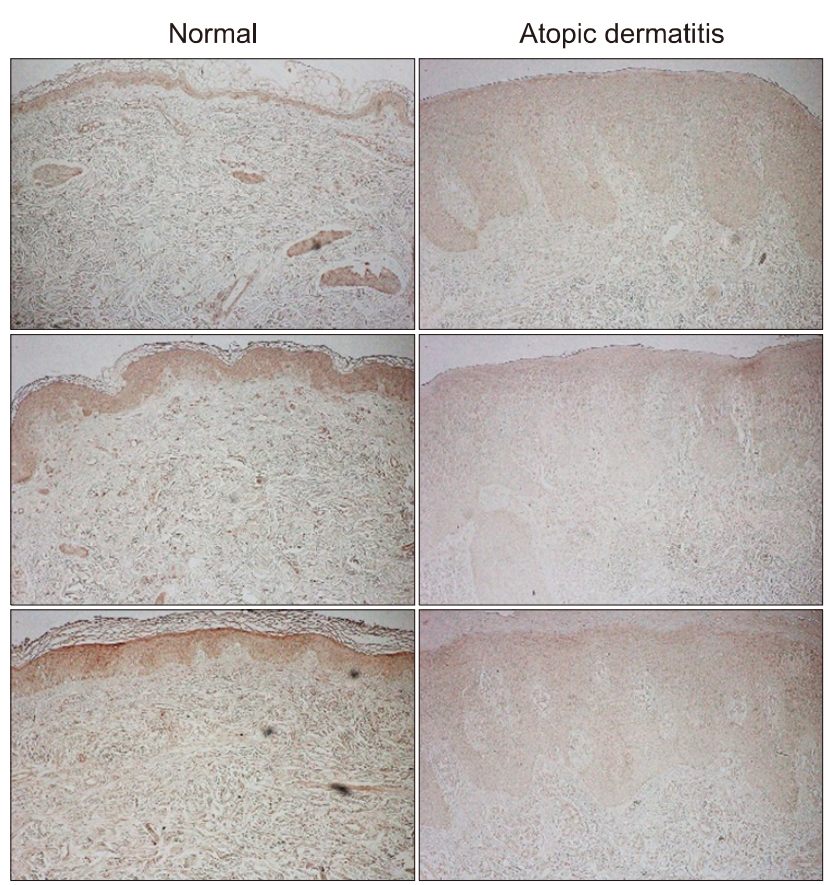

B

Normal Atopic dermatitis

Fig. 2. Immunohistochemical staining of normal skin and atopic dermatitis lesional skin for adiponectin receptors. Protein expression of (A) adiponectin receptor-1 (AdipoR1) and (B) -2 (AdipoR2) in human epidermis were diminished in 3 atopic dermatitis patients, compared to control epidermis from 3 normal young adults ( $\mathrm{n}=3$ in each group). Bar=100 $\mu \mathrm{m}$.

\section{DISCUSSION}

The present study was conducted to investigate the effects of adiponectin on Th2 inflammatory cytokine-induced 3D HEEs. The results revealed that adiponectin inhibits the expression of major inflammatory mediators from keratinocytes, enhances lipid synthesis ability, and promotes epidermal differentiation, which might help to improve AD. CAIl is involved in the maintenance of cellular $\mathrm{pH}$, water transport, and ion homeostasis; therefore, it could also contribute to pathological changes that are characteristic for AD, such as spongiosis ${ }^{23}$. NELL2 is a neural tissue-specific epidermal growth factor-like repeat domain-containing protein ${ }^{22}$. The pathophysiological role of NELL2 in AD has not been elucidated. However, it is known that the expression of NELL2 is elevated by Th2 cytokines (IL-4 and IL-13) similar to CAII expression and is specifically increased in AD lesions ${ }^{22,24}$. Thus, CAll and NELL2 are considered molecular epidermal markers of $\mathrm{AD}^{25}$.

The 3D model used in this study was established according to the existing model described in previous studies, which showed that the increase of CAIl and NELL2 mRNA is a relatively specific event for $A D$ epidermal inflammation ${ }^{6,21-23}$. Similar to the observations documented in these previous reports, we also observed an increase of inflammatory cytokines related to AD, such as TSLP and IL-8, and the increase of CAII and NELL2, as well as AD-associated histopathological changes in our 3D model. Therefore, we reconstructed the AD-like inflammatory conditions in our in vitro experiments.

Adiponectin exerts anti-inflammatory actions on several cell types including macrophages, endothelial cells, cardiomyocytes, and fibroblasts 9 . Adiponectin reduces lipopolysaccharide-induced expression of TNF- $\alpha$ in cultured macrophages through the inhibition of nuclear factor- $\kappa \mathrm{B}$ $(\mathrm{NF}-\kappa \mathrm{B})$ signaling and also exhibits anti-inflammatory effects via the direct modulation of the macrophage phenotype ${ }^{26-28}$. The expression of TNF- $\alpha$ and matrix metalloproteinase-12 in macrophages in the lung decreased by adiponectin treatment and the infiltration of CD4 + T lymphocytes into atherosclerotic lesions was attenuated via the suppression of $\mathrm{T}$ cell chemoattractants in macrophages ${ }^{29,30}$. In endothelial cells, adiponectin also attenuates the inflammatory response in vascular walls by reducing adhesion molecules and IL- 6 expression ${ }^{31}$. Furthermore, adiponectin attenuates TNF- $\alpha$ expression in cardiomyocytes and fibroblasts, leading to reduced cellular apopto$\mathrm{sis}^{32}$. Therefore, adiponectin has a protective effect on various organs, including the heart, vasculature, and lung, by balancing the excessive inflammatory response, such as the inhibition of TNF- $\alpha$ induced NF- $\kappa$ B activation.

In the case of psoriasis, a typical chronic inflammatory 

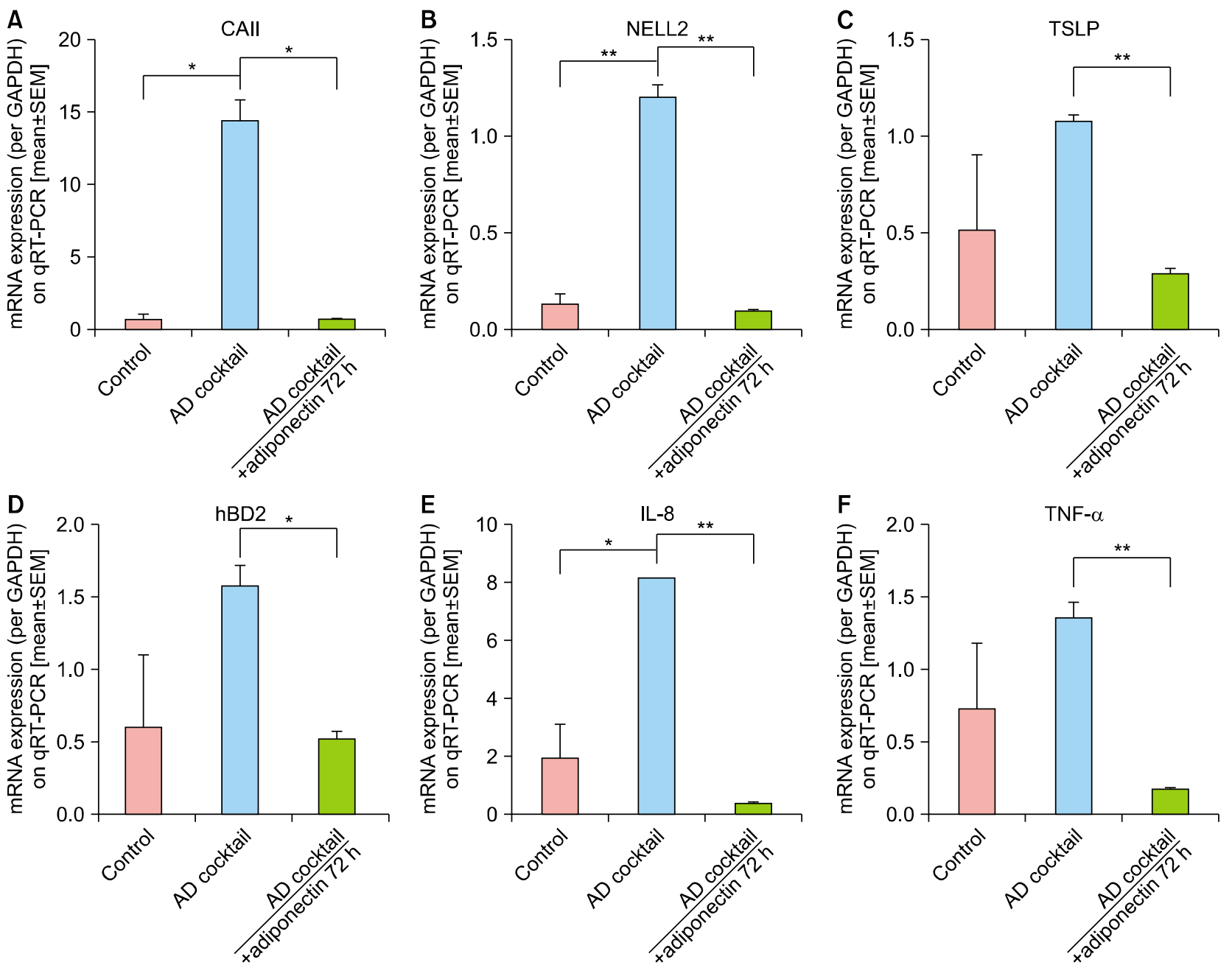

Fig. 3. Effects of adiponectin on inflammatory markers in three-dimensional skin atopic dermatitis (AD) model. The quantitative real-time reverse transcriptase polymerase chain reaction analysis showed the changes in the expression of major inflammatory markers by adiponectin treatment. (A) carbonic anhydrase II (CAII), (B) neuron-specific NEL-like protein 2 (NELL2), (C) thymic stromal lymphopoietin (TSLP) were evaluated as the specific inflammatory markers for atopic dermatitis. Others, (D) human beta-defensin 2 (hBD2), (E) interleukin (IL)-8, and (F) tumor necrosis factor-alpha (TNF- $\alpha$ ) were assessed as the primary inflammatory markers. ${ }^{*} p<0.05, * * p<0.01$.
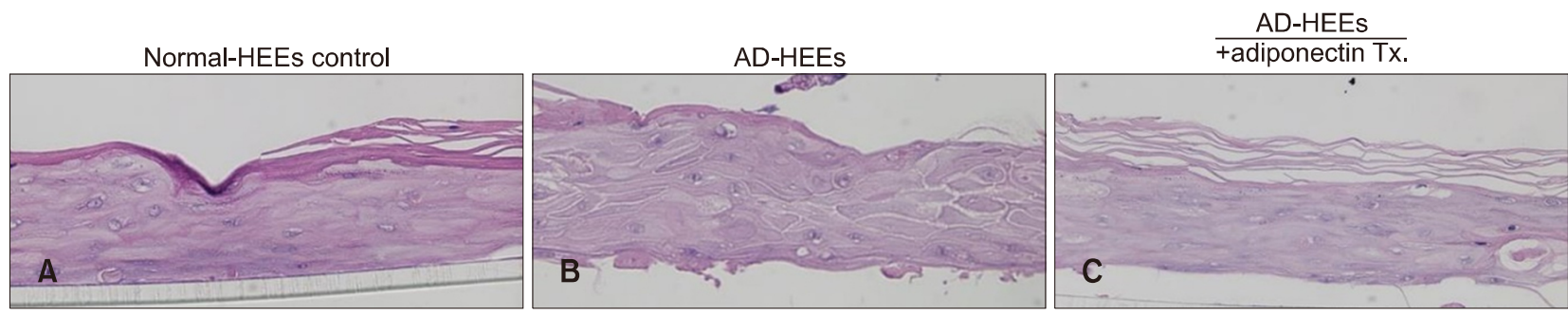

Fig. 4. Representative microscopic finding of (A) normal untreated control, (B) control for reconstructed epidermal atopic dermatitis (AD) model (only the inflammatory cocktail treated), and (C) the adiponectin treated sample (the inflammatory cocktail and adiponectin co-treated). HEEs: human epidermal equivalents, Tx.: treatment.

skin disease, serum adiponectin levels were found to be inversely correlated with disease severity in patients with psoriasis $^{33,34}$. This finding is convincing while considering a series of reports that suggest psoriasis is associated with metabolic syndromes as well as reports of low blood adiponectin levels in obese individuals and patients with dia- 

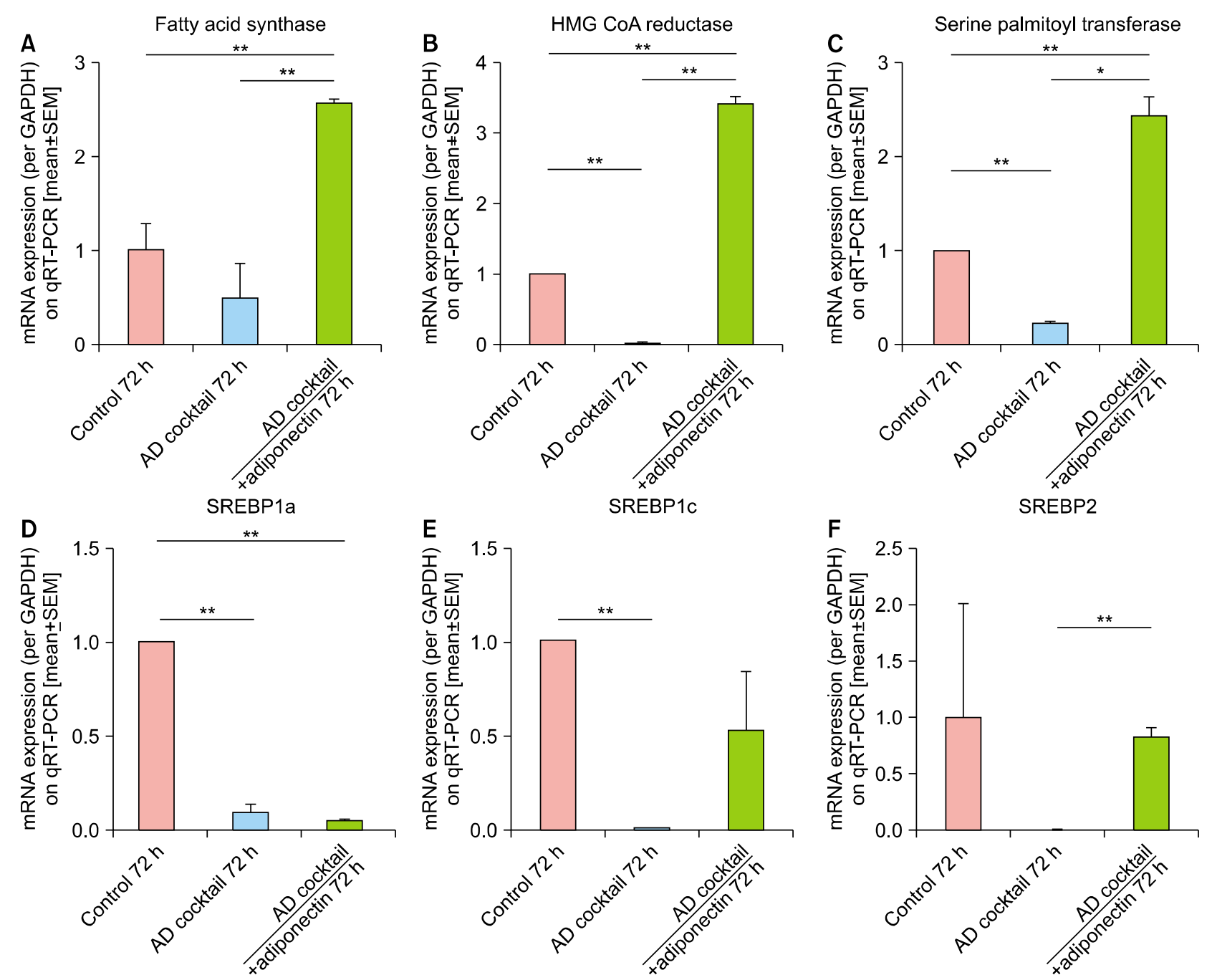

Fig. 5. Effects of adiponectin on expression of lipid synthesis related gene in atopic dermatitis reconstructed epidermal model. By using the quantitative real-time reverse transcriptase polymerase chain reaction analysis, major rate-limiting epidermal lipid biosynthesis enzymes (A) fatty acid synthase, (B) HMG CoA reductase, and (C) serine palimitoyl transferase, and the transcription factors encoding for lipid synthesis enzymes (D) sterol regulatory binding protein 1a (SREBP1a), (E) SREBP1c, and (F) SREBP2 were assessed. ${ }^{*} p<0.05$, $* * p<0.01$.

betes $^{19}$. Moreover, it has been recently reported that adiponectin level in the skin tissue and subcutaneous fat decreased in patients with psoriasis, and adiponectin regulates psoriasis-induced skin inflammation by suppressing IL-17 production from human CD4 + or CD8 + T cells ${ }^{17}$. These observations suggested that adiponectin signaling is a candidate mechanism to explain the relationship between psoriasis and metabolic disorders, and it plays a role in controlling cutaneous inflammation.

In relation to allergic inflammatory disorders, including $A D$, interest in adipokines is increasing in an effort to identify the mechanism of increased prevalence or severity of metabolic problems, such as obesity, as in psoriasis. The serum and tissue transcriptional levels of adiponectin were lower in patients with AD (especially in patients with extrinsic $A D$ ) than in normal subjects, and they were inversely correlated with the total IgE level ${ }^{35-37}$. Furthermore, in asthma, an airway allergic condition, lower levels of adiponectin were associated with uncontrolled or greater risk for asthma ${ }^{38,39}$. However, there is also a report that implicates the negative effects, that is, adiponectin does not protect against the development of inflammation in asthma and might exacerbate the disease via its anti-TH1 inflammatory effects, allowing increased $\mathrm{TH} 2$ differentiation and more severe allergic response ${ }^{40}$. Although there is only limited data available to exactly determine the effect of adiponectin, considering the present results that the expression levels of inflammatory mediators, such as TSLP, 


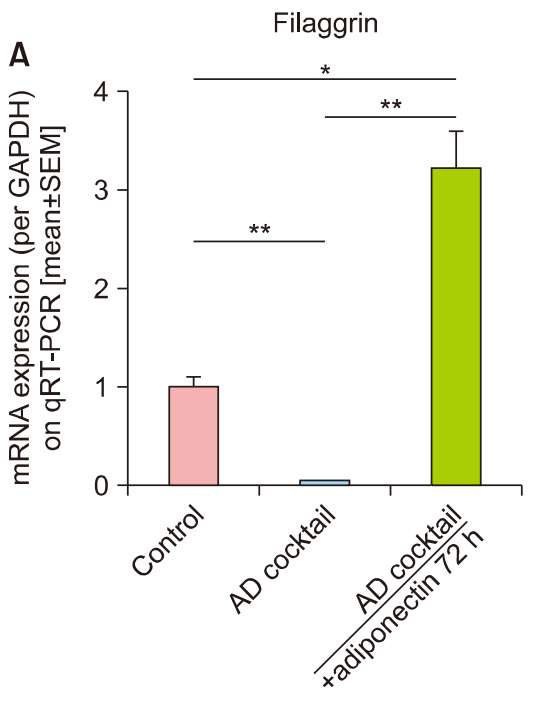

D

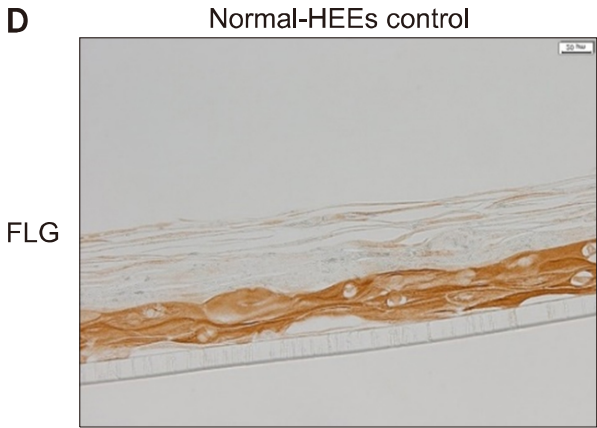

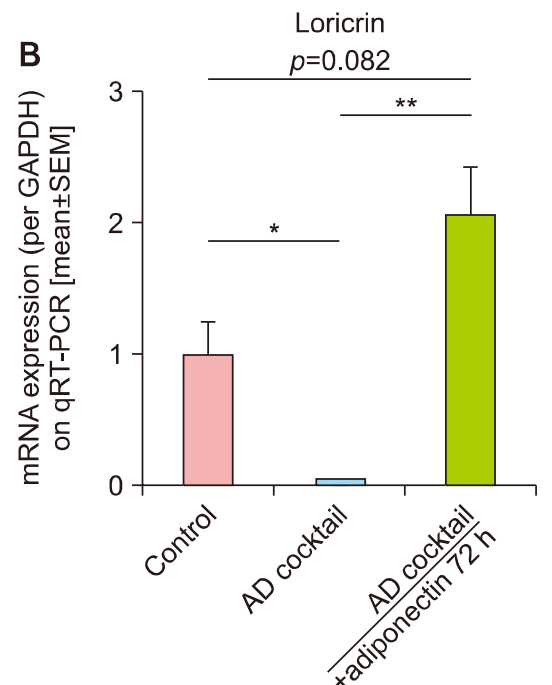

AD-HEEs

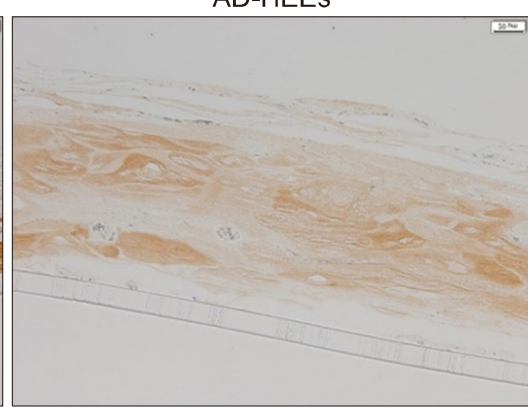

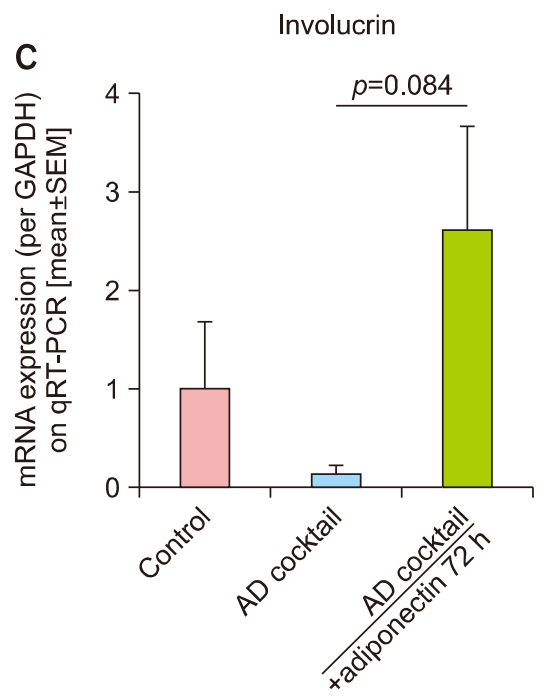

AD-HEEs

+adiponectin Tx.

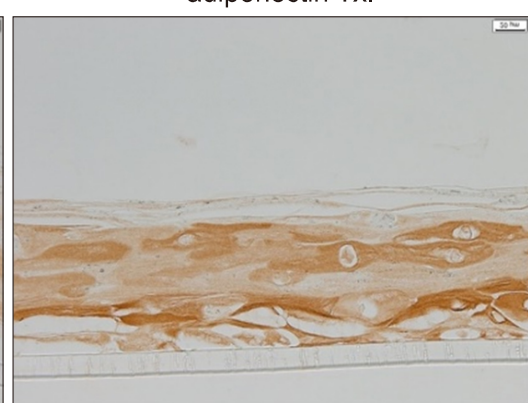

Fig. 6. Effects of adiponectin on epidermal differentiation in atopic dermatitis (AD) reconstructed epidermal model. The mRNA expression of the major epidermal differentiation markers (A) filaggrin (FLG), (B) loricrin, and (C) involucrin was quantified using real time reverse transcriptase polymerase chain reaction. (D) Representative immunohistochemical staining of untreated normal epidermal equivalent (normal-HEEs), and AD mimicking epidermis equivalent (AD-HEEs), and adiponectin treated AD mimicking equivalent (AD-HEEs + adiponectin) with human filaggrin antibody. Tx.: treatment. Bar $=50 \mu \mathrm{m} .{ }^{*} p<0.05,{ }^{* *} p<0.01$.

IL-8, and TNF- $\alpha$, were reduced in AD-HEEs by adiponectin treatment, it can be assumed that the normalization of the serum adiponectin level by lowering its level in obese patients by weight control might improve skin inflammation and epidermal barrier function.

We also found decreased expression of adiponectin receptors in the epidermis of patients with $A D$, which might result from the suppression exerted by the inflammatory cytokines. If this is true, further studies are necessary to confirm whether the reduction in skin inflammation severity by anti-inflammatory agents leads to the normalization of receptor expression.

Adiponectin increased the mRNA expression of differentiation proteins (filaggrin, loricrin, and involucrin) and the major epidermal lipid biosynthetic enzymes (FAS, HMGCR, and SPT) in the AD-like epidermis. The differentiation promotion, especially the increase in filaggrin expression is consistent with the findings of previous studies that adiponectin directly upregulated filaggrin expres- sion in normal human epidermal keratinocytes through the sirtuin 1-mediated pathway ${ }^{41,42}$.

Accordingly, for an effective AD therapy, it is expected that the activation of the adiponectin pathway might enhance epidermal differentiation and barrier function as well as attenuate the inflammatory response of keratinocytes. Therefore, the development of an adiponectin-like agonist of adiponectin signals or receptors might be beneficial for the treatment of AD. Further studies are needed to validate our findings and elucidate the underlying mechanisms.

\section{ACKNOWLEDGMENT}

This study was supported by a grant of the Korea Healthcare technology R\&D Project, Ministry of Health \& Welfare, Republic of Korea (Grant no. HN14C0095). 


\section{CONFLICTS OF INTEREST}

The authors have nothing to disclose.

\section{ORCID}

Hee-Seok Seo, https://orcid.org/0000-0001-9493-2885

Ki Hyun Seong, https://orcid.org/0000-0002-8425-837X

Chang-Deok Kim, https://orcid.org/0000-0001-9341-6491

Seong Jun Seo, https://orcid.org/0000-0003-2915-839X

Byung Cheol Park, https://orcid.org/0000-0002-8975-2051

Myung Hwa Kim, https://orcid.org/0000-0002-9072-201X

Seung-Phil Hong, https://orcid.org/0000-0002-0684-498X

\section{REFERENCES}

1. Elias PM, Wakefield JS. Mechanisms of abnormal lamellar body secretion and the dysfunctional skin barrier in patients with atopic dermatitis. J Allergy Clin Immunol 2014;134: 781-791.e1.

2. Berdyshev E, Goleva E, Bronova I, Dyjack N, Rios C, Jung J, et al. Lipid abnormalities in atopic skin are driven by type 2 cytokines. JCI Insight 2018;3:98006.

3. Matsumura S, Terao M, Murota H, Katayama I. Th2 cytokines enhance TrkA expression, upregulate proliferation, and downregulate differentiation of keratinocytes. J Dermatol Sci 2015;78:215-223.

4. Ong PY, Ohtake T, Brandt C, Strickland I, Boguniewicz M, Ganz T, et al. Endogenous antimicrobial peptides and skin infections in atopic dermatitis. N Engl J Med 2002;347: 1151-1160.

5. Bernard FX, Morel F, Camus M, Pedretti N, Barrault C, Garnier J, et al. Keratinocytes under fire of proinflammatory cytokines: bona fide innate immune cells involved in the physiopathology of chronic atopic dermatitis and psoriasis. J Allergy (Cairo) 2012;2012:718725.

6. Rouaud-Tinguely P, Boudier D, Marchand L, Barruche V, Bordes $\mathrm{S}$, Coppin $\mathrm{H}$, et al. From the morphological to the transcriptomic characterization of a compromised threedimensional in vitro model mimicking atopic dermatitis. $\mathrm{Br}$ J Dermatol 2015;173:1006-1014.

7. Jiang WG, Sanders AJ, Ruge F, Harding KG. Influence of interleukin-8 (IL-8) and IL-8 receptors on the migration of human keratinocytes, the role of PLC- $\gamma$ and potential clinical implications. Exp Ther Med 2012;3:231-236.

8. Jung YR, Lee JH, Sohn KC, Lee Y, Seo YJ, Kim CD, et al. Adiponectin signaling regulates lipid production in human sebocytes. PLoS One 2017; 12:e0169824.

9. Ohashi K, Shibata R, Murohara T, Ouchi N. Role of antiinflammatory adipokines in obesity-related diseases. Trends Endocrinol Metab 2014;25:348-355.

10. Yamauchi T, Kadowaki T. Adiponectin receptor as a key player in healthy longevity and obesity-related diseases. Cell Metab 2013;17:185-196.

11. Kadowaki T, Yamauchi T, Okada-Iwabu M, Iwabu M. Adi- ponectin and its receptors: implications for obesity-associated diseases and longevity. Lancet Diabetes Endocrinol 2014;2:8-9.

12. Jin T, Kim MJ, Heo WI, Park KY, Choi SY, Lee MK, et al. Adiponectin corrects premature cellular senescence and normalizes antimicrobial peptide levels in senescent keratinocytes. Biochem Biophys Res Commun 2016;477:678-684.

13. Cnop M, Havel PJ, Utzschneider KM, Carr DB, Sinha MK, Boyko EJ, et al. Relationship of adiponectin to body fat distribution, insulin sensitivity and plasma lipoproteins: evidence for independent roles of age and sex. Diabetologia 2003;46:459-469.

14. Lago F, Dieguez C, Gómez-Reino J, Gualillo O. Adipokines as emerging mediators of immune response and inflammation. Nat Clin Pract Rheumatol 2007;3:716-724.

15. van Stijn CM, Kim J, Barish GD, Tietge UJ, Tangirala RK. Adiponectin expression protects against angiotensin II-mediated inflammation and accelerated atherosclerosis. PLoS One 2014;9:e86404.

16. Fasshauer $\mathrm{M}$, Blüher $\mathrm{M}$. Adipokines in health and disease. Trends Pharmacol Sci 2015;36:461-470.

17. Shibata S, Tada Y, Hau CS, Mitsui A, Kamata M, Asano Y, et al. Adiponectin regulates psoriasiform skin inflammation by suppressing IL-17 production from $\gamma \delta-T$ cells. Nat Commun 2015;6:7687.

18. Campanati A, Ganzetti G, Giuliodori K, Marra M, Bonfigli A, Testa R, et al. Serum levels of adipocytokines in psoriasis patients receiving tumor necrosis factor- $a$ inhibitors: results of a retrospective analysis. Int J Dermatol 2015;54:839-845.

19. Prussick RB, Miele L. Nonalcoholic fatty liver disease in patients with psoriasis: a consequence of systemic inflammatory burden? Br J Dermatol 2018;179:16-29.

20. Danso MO, van Drongelen V, Mulder A, van Esch J, Scott $\mathrm{H}$, van Smeden J, et al. TNF-a and Th2 cytokines induce atopic dermatitis-like features on epidermal differentiation proteins and stratum corneum lipids in human skin equivalents. J Invest Dermatol 2014;134:1941-1950.

21. Kamsteeg M, Bergers $M$, de Boer R, Zeeuwen PL, Hato SV, Schalkwijk J, et al. Type 2 helper T-cell cytokines induce morphologic and molecular characteristics of atopic dermatitis in human skin equivalent. Am J Pathol 2011;178: 2091-2099.

22. Nomura I, Gao B, Boguniewicz M, Darst MA, Travers JB, Leung DY. Distinct patterns of gene expression in the skin lesions of atopic dermatitis and psoriasis: a gene microarray analysis. J Allergy Clin Immunol 2003;112:1195-1202.

23. Kamsteeg M, Zeeuwen PL, de Jongh GJ, Rodijk-Olthuis D, Zeeuwen-Franssen ME, van Erp PE, et al. Increased expression of carbonic anhydrase II (CA II) in lesional skin of atopic dermatitis: regulation by Th2 cytokines. J Invest Dermatol 2007;127:1786-1789.

24. Kamsteeg $M$, Jansen PA, van Vlijmen-Willems IM, van Erp PE, Rodijk-Olthuis D, van der Valk PG, et al. Molecular diagnostics of psoriasis, atopic dermatitis, allergic contact dermatitis and irritant contact dermatitis. $\mathrm{Br} J$ Dermatol 2010; 162:568-578.

25. van den Bogaard EH, Tjabringa GS, Joosten I, Vonk-Bergers 
M, van Rijssen E, Tijssen HJ, et al. Crosstalk between keratinocytes and $\mathrm{T}$ cells in a $3 \mathrm{D}$ microenvironment: $\mathrm{a}$ model to study inflammatory skin diseases. J Invest Dermatol 2014;134:719-727.

26. Yokota T, Oritani K, Takahashi I, Ishikawa J, Matsuyama A, Ouchi N, et al. Adiponectin, a new member of the family of soluble defense collagens, negatively regulates the growth of myelomonocytic progenitors and the functions of macrophages. Blood 2000;96:1723-1732.

27. Yamaguchi N, Argueta JG, Masuhiro $Y$, Kagishita $M$, Nonaka K, Saito T, et al. Adiponectin inhibits Toll-like receptor family-induced signaling. FEBS Lett 2005;579: 6821-6826.

28. Ohashi K, Parker JL, Ouchi N, Higuchi A, Vita JA, Gokce N, et al. Adiponectin promotes macrophage polarization toward an anti-inflammatory phenotype. J Biol Chem 2010; 285:6153-6160.

29. Summer R, Little FF, Ouchi N, Takemura Y, Aprahamian T, Dwyer D, et al. Alveolar macrophage activation and an emphysema-like phenotype in adiponectin-deficient mice. Am J Physiol Lung Cell Mol Physiol 2008;294:L1035-L1042.

30. Okamoto Y, Folco EJ, Minami M, Wara AK, Feinberg MW, Sukhova GK, et al. Adiponectin inhibits the production of CXC receptor 3 chemokine ligands in macrophages and reduces T-lymphocyte recruitment in atherogenesis. Circ Res 2008;102:218-225.

31. Komura N, Maeda N, Mori T, Kihara S, Nakatsuji H, Hirata A, et al. Adiponectin protein exists in aortic endothelial cells. PLoS One 2013;8:e71271.

32. Shibata R, Sato K, Pimentel DR, Takemura Y, Kihara S, Ohashi K, et al. Adiponectin protects against myocardial ischemia-reperfusion injury through AMPK- and COX-2dependent mechanisms. Nat Med 2005;11:1096-1103.

33. Bai F, Zheng W, Dong Y, Wang J, Garstka MA, Li R, et al. Serum levels of adipokines and cytokines in psoriasis patients: a systematic review and meta-analysis. Oncotarget
2018;9:1266-1278.

34. Sereflican B, Goksugur N, Bugdayci G, Polat M, Haydar Parlak A. Serum visfatin, adiponectin, and tumor necrosis factor alpha (TNF- $\alpha$ ) levels in patients with psoriasis and their correlation with disease severity. Acta Dermatovenerol Croat 2016;24:13-19.

35. Han B, Wu WH, Bae JM, Son SJ, Lee JH, Han TY. Serum leptin and adiponectin levels in atopic dermatitis (AD) and their relation to disease severity. J Am Acad Dermatol 2016; 75:629-631.

36. Plager DA, Leontovich AA, Henke SA, Davis MD, McEvoy MT, Sciallis GF 2nd, et al. Early cutaneous gene transcription changes in adult atopic dermatitis and potential clinical implications. Exp Dermatol 2007;16:28-36.

37. Guttman-Yassky E, Suárez-Fariñas M, Chiricozzi A, Nograles KE, Shemer A, Fuentes-Duculan J, et al. Broad defects in epidermal cornification in atopic dermatitis identified through genomic analysis. J Allergy Clin Immunol 2009;124:12351244.e1258.

38. Sood A, Shore SA. Adiponectin, leptin, and resistin in asthma: basic mechanisms through population studies. J Allergy (Cairo) 2013;2013:785835.

39. Dogru M, Ozde S, Aktas A, Yuksel Karatoprak E. The adiponectin levels and asthma control in non-obese children with asthma. J Asthma 2015;52:772-776.

40. de Lima Azambuja R, da Costa Santos Azambuja LS, Costa C, Rufino R. Adiponectin in asthma and obesity: protective agent or risk factor for more severe disease? Lung 2015; 193:749-755.

41. Jin T, Park KY, Seo SJ. Adiponectin upregulates filaggrin expression via SIRT1-mediated signaling in human normal keratinocytes. Ann Dermatol 2017;29:407-413.

42. Shibata S, Tada Y, Asano Y, Hau CS, Kato T, Saeki H, et al. Adiponectin regulates cutaneous wound healing by promoting keratinocyte proliferation and migration via the ERK signaling pathway. J Immunol 2012;189:3231-3241. 